\title{
Influence of Rotation and Magnetic Fields on a Functionally Graded Thermoelastic Solid Subjected to a Mechanical Load
}

\author{
Ankush Gunghas, ${ }^{1}$ Rajesh Kumar, ${ }^{2}$ Sunita Deswal $\mathbb{D}^{,},{ }^{1}$ and Kapil Kumar Kalkal $\mathbb{D D}^{1}$ \\ ${ }^{1}$ Department of Mathematics, Guru Jambheshwar University of Science and Technology, Hisar 125001, Haryana, India \\ ${ }^{2}$ Department of Applied Sciences, School of Engineering and Technology, The NorthCap University, Gurgaon 122017, Haryana, India
}

Correspondence should be addressed to Kapil Kumar Kalkal; kapilkalkal_gju@rediffmail.com

Received 26 February 2019; Accepted 22 April 2019; Published 10 June 2019

Academic Editor: Viliam Makis

Copyright (C) 2019 Ankush Gunghas et al. This is an open access article distributed under the Creative Commons Attribution License, which permits unrestricted use, distribution, and reproduction in any medium, provided the original work is properly cited.

\begin{abstract}
The current manuscript is presented to study two-dimensional deformations in a nonhomogeneous, isotropic, rotating, magnetothermoelastic medium in the context of Green-Naghdi model III. It is assumed that the functionally graded material has nonhomogeneous mechanical and thermal properties in the $x$-direction. The exact expressions for the displacement components, temperature field, and stresses are obtained in the physical domain by using normal mode technique. These are also computed numerically for a copper-like material and presented graphically to observe the variations of the considered physical variables. Comparisons of the physical quantities are shown in figures to depict the effects of angular velocity, nonhomogeneity parameter, and magnetic field.
\end{abstract}

\section{Introduction}

In the classical dynamical coupled theory of thermoelasticity formulated by Biot [1], thermal signals are propagating with infinite speed, which is not physically acceptable. To remove this drawback of the classical coupled dynamical theory of thermoelasticity, several theories of generalized thermoelasticity were developed. The first two generalized thermoelastic theories are Lord-Shulman (L-S) [2] theory and Green-Lindsay (G-L) [3] theory. In L-S theory, one thermal relaxation time parameter is introduced in the classical Fourier's law of heat conduction, whereas in the G-L theory, two thermal relaxation times are introduced in the constitutive relations for force stress tensor and entropy equation. Green and Naghdi [4-6] speculated three new thermoelasticity theories that permit treatment of a much wider class of heat flow problems, labeled as G-N models I, II, and III. The nature of the constitutive equations in these three models is such that when the respective theories are linearized, the model I is same as the classical heat conduction theory, model II predicts the finite speed of heat propagation involving no energy dissipation, and model III indicates the propagation of thermal signals with finite speed.

The theory of magneto-thermoelasticity is concerned with the effect of magnetic field on elastic and thermoelastic deformations of a solid body and has received the attention of many researchers due to its extensive use in various fields like optics, geophysics, and acoustics. The problem of distribution of thermal stresses and temperature in a perfectly conducting half-space, in contact with a vacuum, permeated by an initial magnetic field was studied by Ezzat [7]. A model of two-dimensional equations of generalized magneto-thermoelasticity with one relaxation time in a perfectly conducting medium was established by Ezzat and Othman [8]. The impacts of fractional order parameter, hydrostatic initial stress, and gravity field on the plane waves in a fiber-reinforced isotropic thermoelastic medium were investigated by Othman et al. [9]. Deswal and Kalkal [10] employed Laplace and Fourier transforms technique to study the phenomenon of wave propagation in a fractional order micropolar magneto-thermoelastic half-space. In the frame of fractional order theory of generalized thermoelasticity, 
Deswal et al. [11] studied magneto-thermoelastic interactions in an initially stressed, isotropic, homogeneous halfspace. The effects of initial stress and magnetic field on thermoelastic interactions in an isotropic, thermally and electrically conducting half-space, whose surface is subjected to mechanical and thermal loads, were explored by Othman and Eraki [12]. Xiong and Guo [13] investigated the electromagneto-thermoelastic diffusive plane waves in a half-space with variable material properties under fractional order thermoelastic theory.

Since the large bodies like the earth, the moon, and other planets have an angular velocity, it appears more realistic to study the thermoelastic problems in a rotating medium. The propagation of elastic waves in a rotating, homogeneous, and isotropic medium was investigated by Schoenberg and Censor [14]. Some results in thermoelastic rotating medium are due to Roy Choudhuri and Debnath [15] and Roy Choudhuri and Mukhopadhyay [16]. The effect of rotation in generalized thermoelastic solid under the influence of gravity with an overlying infinite thermoelastic fluid was analyzed by Ailawalia and Narah [17]. Abouelregal and Zenkour [18] used fractional order theory of thermoelasticity to scrutinize the effect of angular velocity on fiberreinforced generalized thermoelastic medium whose surface is subjected to a Mode-I crack problem. Kumar et al. [19] discussed the propagation of plane waves at the free surface of thermally conducting micropolar elastic half-space with two temperatures. Othman et al. [20] considered the dual-phase lag model to study the influence of the rotation on a twodimensional problem of micropolar thermoelastic isotropic medium with two temperatures. Said et al. [21] used normal mode technique to study thermodynamical interactions in a micropolar magneto-elastic medium with rotation and twotemperature. Abouelregal and Abo-Dahab [22] investigated a two-dimensional problem in the context of dual-phase-lag model with fiber-reinforcement and rotation using normal mode analysis. The effect of angular velocity on Rayleigh wave propagation in a fiber-reinforced, anisotropic magnetothermo-viscoelastic media was discussed by Hussien and Bayones [23].

Over the last few decades, some structural materials such as functionally graded materials have been rapidly developed and used in many engineering applications. In functionally graded materials (FGMs), material properties vary gradually with a location within the body. FGMs are usually designed to be used under high-temperature environments. So, FGMs can easily eliminate or control thermal stresses, when sudden heating or cooling happens. These types of material are broadly used in important structures such as body materials in the aerospace field and nuclear reactors. A thermoinelastic response of functionally graded composites was studied by Aboudi et al. [24]. Abd-Alla et al. [25] analyzed radial vibrations in a functionally graded orthotropic elastic half-space subjected to rotation and gravity field. The electro-magnetothermoelastic response of an infinite functionally graded cylinder was studied by Abbas and Zenkour [26], by using finite element method. The problem of generalized thermoelasticity in a thick-walled functionally graded cylinder with one relaxation time was considered by Abbas [27]. In this problem, the effects of temperature-dependent properties, volume fraction parameter, and thermal relaxation time on thermophysical quantities are estimated.

The aim of the present contribution is to consider twodimensional disturbances in an infinite, isotropic, nonhomogeneous, rotating, magneto-thermoelastic medium in the context of G-N model III. All the mechanical and thermal properties of the FGM under consideration are supposed to vary as an exponential power of the space-coordinate. The numerical results for the physical quantities have been obtained for a copper-like material and presented graphically to estimate and highlight the effects of different parameters considered in this problem.

\section{Basic Equations}

Following Green-Naghdi [5] and Roy Choudhuri and Debnath [15], the field equations and stress-strain-temperature relations in a rotating thermoelastic medium in the presence of body forces $F_{i}$ are

\section{Constitutive Law}

$$
\sigma_{i j}=2 \mu e_{i j}+\delta_{i j}\left(\lambda e_{r r}-\beta \theta\right)
$$

where

$$
e_{i j}=\frac{1}{2}\left(u_{i, j}+u_{j, i}\right)
$$

Stress Equation of Motion

$$
\begin{aligned}
\sigma_{j i, j} & +F_{i} \\
& =\rho\left(\frac{\partial^{2} u_{i}}{\partial t^{2}}+(\vec{\Omega} \times(\vec{\Omega} \times \vec{u}))_{i}+(2 \vec{\Omega} \times \dot{\vec{u}})_{i}\right) .
\end{aligned}
$$

Equation of Heat Conduction

$$
K^{*} \nabla^{2} \theta+K \nabla^{2} \dot{\theta}=\rho C_{e} \ddot{\theta}+\beta T_{0} \ddot{e}
$$

where $\lambda, \mu$ are Lame's elastic constants, $\beta=(3 \lambda+2 \mu) \alpha_{t}$, $\alpha_{t}$ is the coefficient of linear thermal expansion, $\sigma_{i j}$ are the components of stress, $e_{i j}$ are the components of strain, $\rho$ is the reference mass density, $C_{e}$ is the specific heat at constant strain, $K$ is the thermal conductivity, $K^{*}$ is the material constant characteristic for this theory, $\vec{u}$ is the displacement vector, $\theta=T-T_{0}, T$ is the absolute temperature, $T_{0}$ is the reference temperature of the medium in its natural state assumed to be $\left|\theta / T_{0}\right| \ll 1, e=e_{r r}$ is the cubical dilatation, $\vec{\Omega}$ is the rotation vector, and $\delta_{i j}$ is the Kronecker delta.

For a nonhomogeneous medium, the parameters $\lambda, \mu$, $\beta, K, K^{*}$, and $\rho$ are no longer constant but become spacedependent. Hence we replace $\lambda, \mu, \beta, K, K^{*}$, and $\rho$ by $\lambda_{0} f(\vec{x}), \mu_{0} f(\vec{x}), \beta_{0} f(\vec{x}), K_{0} f(\vec{x}), K_{0}^{*} f(\vec{x})$, and $\rho_{0} f(\vec{x})$, respectively, where $\lambda_{0}, \mu_{0}, \beta_{0}, K_{0}, K_{0}^{*}$, and $\rho_{0}$ are supposed to be constants and $f(\vec{x})$ is a given nondimensional function 
of the space variable $\vec{x}=(x, y, z)$. Using these values of parameters, (1), (3), and (4) take the following form:

$$
\begin{aligned}
& \sigma_{i j}=f(\vec{x})\left[2 \mu_{0} e_{i j}+\delta_{i j}\left(\lambda_{0} e_{r r}-\beta_{0} \theta\right)\right], \\
& \sigma_{j i, j}+F_{i}=\rho_{0} f(\vec{x}) \\
& \cdot\left(\frac{\partial^{2} u_{i}}{\partial t^{2}}+(\vec{\Omega} \times(\vec{\Omega} \times \vec{u}))_{i}+(2 \vec{\Omega} \times \dot{\vec{u}})_{i}\right), \\
&\left(K_{0}^{*} f(\vec{x}) \theta_{,_{i}}\right)_{i_{i}}+\left(K_{0} f(\vec{x}) \dot{\theta}_{,_{i}}\right)_{, i}=f(\vec{x}) \\
& \cdot\left(\rho_{0} C_{e} \ddot{\theta}+\beta_{0} T_{0} \ddot{e}\right),
\end{aligned}
$$

where

$$
\beta_{0}=\left(3 \lambda_{0}+2 \mu_{0}\right) \alpha_{t}
$$

Here, the superposed dot denotes derivative with respect to time and the comma denotes derivative with respect to space variable.

\section{Mathematical Model}

Consider a nonhomogeneous, isotropic, magneto-thermoelastic half-space under the purview of G-N model III. Rectangular Cartesian coordinates are introduced having the surface of the half-space as the plane $x=0$, with $x$-axis pointing vertically downwards into the medium. The medium is rotating with an angular velocity $\vec{\Omega}$. Thus the displacement equation of motion in the rotating plane has two extra terms: $\vec{\Omega} \times(\vec{\Omega} \times \vec{u})$, which is the centripetal acceleration due to time varying motion only and $2 \vec{\Omega} \times \dot{\vec{u}}$, which is the Coriolis acceleration. The present formulation is restricted to $x y$-plane and thus all the field variables are independent of the space variable $z$. So the displacement vector $\vec{u}$ and angular velocity $\vec{\Omega}$ will have the components:

$$
\begin{aligned}
& \vec{u}=\left(u_{1}, u_{2}, 0\right), \\
& \vec{\Omega}=(0,0, \Omega) .
\end{aligned}
$$

It is also assumed that material properties are graded only in $x$-direction. So we take $f(\vec{x})$ as $f(x)$. By virtue of (9), the stresses arising from (5) can be expressed as

$$
\begin{aligned}
& \sigma_{x x}=f(x)\left[\left(\lambda_{0}+2 \mu_{0}\right) \frac{\partial u_{1}}{\partial x}+\lambda_{0} \frac{\partial u_{2}}{\partial y}-\beta_{0} \theta\right], \\
& \sigma_{y y}=f(x)\left[\left(\lambda_{0}+2 \mu_{0}\right) \frac{\partial u_{2}}{\partial y}+\lambda_{0} \frac{\partial u_{1}}{\partial x}-\beta_{0} \theta\right], \\
& \sigma_{x y}=f(x) \mu_{0}\left(\frac{\partial u_{1}}{\partial y}+\frac{\partial u_{2}}{\partial x}\right) .
\end{aligned}
$$

Due to the application of an initial magnetic field $\vec{H}\left(0,0, H_{0}\right)$, an induced magnetic field $\vec{h}$, an induced electric field $\vec{E}$, and a current density $\vec{J}$ are developed in the considered medium.
The simplified linear equations of electrodynamics of a slowly moving medium for a nonhomogeneous, isotropic and thermally conducting elastic solid are given by, neglecting Thomson's effect [28]:

$$
\begin{aligned}
\nabla \times \vec{h} & =\vec{J}, \\
\nabla \times \vec{E} & =-\mu_{m} \frac{\partial \vec{h}}{\partial t}, \\
\nabla \cdot \vec{h} & =0, \\
\nabla \cdot \vec{E} & =0, \\
\vec{E} & =-\mu_{m}\left(\frac{\partial \vec{u}}{\partial t} \times \vec{H}\right), \\
\vec{h} & =\nabla \times(\vec{u} \times \vec{H}),
\end{aligned}
$$

where $\mu_{m}$ is the magnetic permeability of the medium.

From the above expressions, one can obtain

$$
\begin{aligned}
& \vec{E}=\left(-\mu_{m} H_{0} \frac{\partial u_{2}}{\partial t}, \mu_{m} H_{0} \frac{\partial u_{1}}{\partial t}, 0\right), \\
& \vec{h}=\left[0,0,-H_{0}\left(\frac{\partial u_{1}}{\partial x}+\frac{\partial u_{2}}{\partial y}\right)\right], \\
& \vec{J}=\left(\frac{\partial h_{3}}{\partial y},-\frac{\partial h_{3}}{\partial x}, 0\right),
\end{aligned}
$$

where

$$
h_{3}=-H_{0}\left(\frac{\partial u_{1}}{\partial x}+\frac{\partial u_{2}}{\partial y}\right)
$$

By virtue of above expressions and replacing $\mu_{m}$ by $\mu_{m 0} f(x)$, the components of the Lorentz force $\vec{F}=\mu_{m}(\vec{J} \times \vec{H})$ are given by

$$
\begin{aligned}
& F_{1}=f(x) \mu_{m 0} H_{0}^{2} \frac{\partial e}{\partial x}, \\
& F_{2}=f(x) \mu_{m 0} H_{0}^{2} \frac{\partial e}{\partial y}, \\
& F_{3}=0 .
\end{aligned}
$$

Utilizing the components of Lorentz force into stress equation of motion along with the consideration of two-dimensional problem, the field equations (6) and (7) yield 


$$
\begin{gathered}
f(x)\left[\left(\lambda_{0}+2 \mu_{0}\right) \frac{\partial^{2} u_{1}}{\partial x^{2}}+\left(\lambda_{0}+\mu_{0}\right) \frac{\partial^{2} u_{2}}{\partial x \partial y}+\mu_{0} \frac{\partial^{2} u_{1}}{\partial y^{2}}\right. \\
\left.-\beta_{0} \frac{\partial \theta}{\partial x}\right]+\frac{\partial f(x)}{\partial x}\left[\left(\lambda_{0}+2 \mu_{0}\right) \frac{\partial u_{1}}{\partial x}+\lambda_{0} \frac{\partial u_{2}}{\partial y}\right. \\
\left.-\beta_{0} \theta\right]+f(x) \mu_{m 0} H_{0}^{2} \frac{\partial e}{\partial x}=f(x) \rho_{0}\left(\frac{\partial^{2} u_{1}}{\partial t^{2}}\right. \\
\left.-\Omega^{2} u_{1}-2 \Omega \frac{\partial u_{2}}{\partial t}\right), \\
f(x)\left[\left(\lambda_{0}+2 \mu_{0}\right) \frac{\partial^{2} u_{2}}{\partial y^{2}}+\left(\lambda_{0}+\mu_{0}\right) \frac{\partial^{2} u_{1}}{\partial x \partial y}+\mu_{0} \frac{\partial^{2} u_{2}}{\partial x^{2}}\right. \\
\left.-\beta_{0} \frac{\partial \theta}{\partial y}\right]+\mu_{0} \frac{\partial f(x)}{\partial x}\left(\frac{\partial u_{1}}{\partial y}+\frac{\partial u_{2}}{\partial x}\right)+f(x) \\
+\mu_{m 0} H_{0}^{2} \frac{\partial e}{\partial y}=f(x) \rho_{0}\left(\frac{\partial^{2} u_{2}}{\partial t^{2}}-\Omega^{2} u_{2}+2 \Omega \frac{\partial u_{1}}{\partial t}\right) \\
K_{0}^{*}\left(f(x) \nabla^{2} \theta+\frac{\partial f(x)}{\partial x} \frac{\partial \theta}{\partial x}\right)+K_{0}\left(f(x) \nabla^{2} \dot{\theta}\right. \\
+(x)
\end{gathered}
$$

where

$$
\nabla^{2}=\frac{\partial^{2}}{\partial x^{2}}+\frac{\partial^{2}}{\partial y^{2}}
$$

\section{Exponential Variation of Nonhomogeneity}

By assuming $f(x)=e^{-n x}$, where $n$ is a dimensionless parameter, one can conclude that the mechanical and thermal properties of the material vary exponentially along the $x$ direction. The governing equations can be recast in the dimensionless form by introducing the following dimensionless parameters:

$$
\begin{aligned}
\left(x^{\prime}, y^{\prime}\right) & =\frac{w^{*}}{c_{1}}(x, y), \\
\left(u_{1}^{\prime}, u_{2}^{\prime}\right) & =\frac{\rho_{0} w^{*} c_{1}}{\beta_{0} T_{0}}\left(u_{1}, u_{2}\right), \\
t^{\prime} & =w^{*} t, \\
\theta^{\prime} & =\frac{\theta}{T_{0}} \\
\sigma_{i j}^{\prime} & =\frac{\sigma_{i j}}{\beta_{0} T_{0}}, \\
\Omega^{\prime} & =\frac{\Omega}{w^{*}},
\end{aligned}
$$

where

$$
\begin{aligned}
w^{*} & =\frac{\rho_{0} C_{e} c_{1}^{2}}{K_{0}}, \\
c_{1}^{2} & =\frac{\lambda_{0}+2 \mu_{0}}{\rho_{0}} .
\end{aligned}
$$

Now, in terms of the dimensionless parameters given in (22), (10)-(12) and (18)-(20) transform to

$$
\begin{aligned}
\sigma_{x x} & =e^{-n x}\left(\frac{\partial u_{1}}{\partial x}+A_{11} \frac{\partial u_{2}}{\partial y}-\theta\right) \\
\sigma_{y y} & =e^{-n x}\left(\frac{\partial u_{2}}{\partial y}+A_{11} \frac{\partial u_{1}}{\partial x}-\theta\right) \\
\sigma_{x y} & =e^{-n x} A_{12}\left(\frac{\partial u_{1}}{\partial y}+\frac{\partial u_{2}}{\partial x}\right) \\
\left(1+A_{22}\right) & \frac{\partial^{2} u_{1}}{\partial x^{2}}+\left(A_{21}+A_{22}\right) \frac{\partial^{2} u_{2}}{\partial x \partial y}+A_{12} \frac{\partial^{2} u_{1}}{\partial y^{2}} \\
& -\frac{\partial \theta}{\partial x}-n\left(\frac{\partial u_{1}}{\partial x}+A_{11} \frac{\partial u_{2}}{\partial y}-\theta\right)=\frac{\partial^{2} u_{1}}{\partial t^{2}}-\Omega^{2} u_{1} \\
& -2 \Omega \frac{\partial u_{2}}{\partial t}, \\
\nabla^{2} \theta & -n \frac{\partial \theta}{\partial x}+A_{31}\left(\nabla^{2} \dot{\theta}-n \frac{\partial \dot{\theta}}{\partial x}\right)=A_{32} \frac{\partial \dot{\theta}}{\partial t}+A_{33} \frac{\partial \dot{e}}{\partial t} \\
& +2 \Omega \frac{\partial u_{1}}{\partial t}, \\
& -\frac{\partial \theta}{\partial y}-n A_{12}\left(\frac{\partial^{2} u_{2}}{\partial y^{2}}+\left(A_{21}+A_{22}\right) \frac{\partial^{2} u_{1}}{\partial x \partial y}+\frac{\partial u_{2}}{\partial x}\right)=\frac{\partial^{2} u_{2}}{\partial t^{2}}-\Omega^{2} u_{2} \\
& +x^{2}
\end{aligned}
$$

where

$$
\begin{aligned}
& A_{11}=\frac{\lambda_{0}}{\rho_{0} c_{1}^{2}}, \\
& A_{12}=\frac{\mu_{0}}{\rho_{0} c_{1}^{2}}, \\
& A_{21}=A_{11}+A_{12}, \\
& A_{22}=\frac{\mu_{m 0} H_{0}^{2}}{\rho_{0} c_{1}^{2}}, \\
& A_{31}=\frac{K_{0} w^{*}}{K_{0}^{*}}, \\
& A_{32}=\frac{\rho_{0} C_{e} c_{1}^{2}}{K_{0}^{*}}, \\
& A_{33}=\frac{\beta_{0}^{2} T_{0}}{\rho_{0} K_{0}^{*}} .
\end{aligned}
$$




\section{Solution Methodology}

In this section, the normal mode method is employed, which has the advantage of finding the exact solutions without any assumed constraints on the field variables. In this approach, the solution of the physical variables is decomposed in terms of normal modes and one gets exact solution without any assumed restrictions on the actual physical quantities that appear in the governing equations of the problem considered. Normal mode analysis is, in fact, to look for the solution in Fourier transform domain. It is assumed that all the functions are sufficiently smooth on the real line such that the normal mode analysis of these functions exists. So, the solution for the considered physical variables can be decomposed in terms of normal modes in the following form:

$$
\begin{aligned}
& {\left[u_{1}, u_{2}, \theta, \sigma_{i j}\right](x, y, t)} \\
& \quad=\left[u_{1}^{*}, u_{2}^{*}, \theta^{*}, \sigma_{i j}^{*}\right](x) \exp (\omega t+\iota m y),
\end{aligned}
$$

where $u_{1}^{*}, u_{2}^{*}, \theta^{*}$, and $\sigma_{i j}^{*}$ are the amplitudes of the functions, $\omega$ is the angular frequency, $\iota$ is the imaginary unit, and $m$ is the wave number in $y$-direction.

Introducing expression (31) in (27)-(29), we get

$$
\begin{aligned}
& \left(B_{11} D^{2}-n D+B_{12}\right) u_{1}^{*}+\left(B_{13} D+B_{14}\right) u_{2}^{*} \\
& \quad-(D-n) \theta^{*}=0 \\
& \left(B_{13} D-B_{15}\right) u_{1}^{*}+\left(A_{12} D^{2}-B_{16} D+B_{17}\right) u_{2}^{*} \\
& \quad+B_{18} \theta^{*}=0 \\
& B_{19} D u_{1}^{*}+B_{21} u_{2}^{*}+\left(B_{22} D^{2}-B_{23} D-B_{24}\right) \theta^{*}=0,
\end{aligned}
$$

where

$$
\begin{aligned}
& B_{11}=1+A_{22}, \\
& B_{12}=\Omega^{2}-A_{12} m^{2}-\omega^{2}, \\
& B_{13}=\left(A_{21}+A_{22}\right) \imath m, \\
& B_{14}=2 \Omega \omega-n A_{11} \iota m, \\
& B_{15}=2 \Omega \omega+n A_{12} \iota m, \\
& B_{16}=n A_{12}, \\
& B_{17}=\Omega^{2}-\omega^{2}-m^{2}\left(1+A_{22}\right), \\
& B_{18}=-\imath m, \\
& B_{19}=-A_{33} \omega^{2}, \\
& B_{21}=-B_{18} B_{19}, \\
& B_{22}=1+A_{31} \omega, \\
& B_{23}=n B_{22}, \\
& B_{24}=m^{2}+A_{31} m^{2} \omega+A_{32} \omega^{2} .
\end{aligned}
$$

The condition for the existence of a nonzero solution of the system of (32)-(34) provides us

$$
\begin{aligned}
& {\left[D^{6}+F_{1} D^{5}+F_{2} D^{4}+F_{3} D^{3}+F_{4} D^{2}+F_{5} D+F_{6}\right]} \\
& \cdot\left(u_{1}^{*}, u_{2}^{*}, \theta^{*}\right)=0,
\end{aligned}
$$

where

$$
\begin{aligned}
F_{1} & =\frac{E_{11} E_{18}+E_{12} E_{17}-A_{12} E_{24}-E_{14} E_{23}}{E_{11} E_{17}-A_{12} E_{23}}, \\
F_{2} & =\frac{E_{11} E_{19}+E_{12} E_{18}+E_{13} F_{17}-E_{25} A_{12}-E_{14} E_{24}-E_{15} E_{23}}{E_{11} E_{17}-A_{12} E_{23}}, \\
F_{3} & =\frac{E_{11} E_{21}+E_{12} E_{19}+E_{13} E_{18}-A_{12} E_{26}-E_{14} E_{25}-E_{15} E_{24}-E_{16} E_{23}}{E_{11} E_{17}-A_{12} E_{23}}, \\
F_{4} & =\frac{E_{11} E_{22}+E_{12} E_{21}+E_{13} E_{19}-E_{14} E_{26}-E_{15} E_{25}-E_{16} E_{24}}{E_{11} E_{17}-A_{12} E_{23}}, \\
F_{5} & =\frac{E_{12} E_{22}+E_{13} E_{21}-E_{15} E_{26}-E_{16} E_{25}}{E_{11} E_{17}-A_{12} E_{23}}, \\
F_{6} & =\frac{E_{13} E_{22}-E_{16} E_{26}}{E_{11} E_{17}-A_{12} E_{23}}, \\
E_{11} & =B_{11} B_{18}+B_{13}, \\
E_{12} & =-\left(n B_{18}+B_{14}+n B_{13}\right), \\
E_{13} & =B_{12} B_{18}+n B_{14}, \\
E_{14} & =-\left(B_{15}+n A_{12}\right),
\end{aligned}
$$




$$
\begin{aligned}
& E_{15}=B_{13} B_{18}+B_{16}+n B_{15}, \\
& E_{16}=B_{14} B_{18}-n B_{16}, \\
& E_{17}=A_{12} B_{22}, \\
& E_{18}=-\left(B_{15} B_{22}+B_{23} A_{12}\right), \\
& E_{19}=B_{16} B_{22}+B_{15} B_{23}-A_{12} B_{24}, \\
& E_{21}=B_{15} B_{24}-B_{16} B_{23}, \\
& E_{22}=-\left(B_{16} B_{24}+B_{18} B_{21}\right), \\
& E_{23}=B_{22} B_{13}, \\
& E_{24}=-\left(B_{13} B_{23}+B_{17} B_{22}\right), \\
& E_{25}=B_{17} B_{23}-B_{13} B_{24}-B_{19} B_{18}, \\
& E_{26}=B_{17} B_{24} .
\end{aligned}
$$

The general solution of (36) which is bounded as $x \longrightarrow \infty$ is given by

$$
\begin{array}{r}
{\left[u_{1}^{*}, u_{2}^{*}, \theta^{*}\right](x)=\sum_{i=1}^{3}\left[1, H_{1 i}, H_{2 i}\right] M_{i}(m, \omega) e^{-\lambda_{i} x},} \\
\text { for } \operatorname{Re}\left(\lambda_{i}\right)>0
\end{array}
$$

where $M_{i}(m, \omega)(i=1,2,3)$ are parameters, depending upon $m$ and $\omega$, and

$$
\begin{aligned}
& H_{1 i}=\frac{E_{11} \lambda_{i}^{2}-E_{12} \lambda_{i}+E_{13}}{A_{12} \lambda_{i}^{3}-E_{14} \lambda_{i}^{2}+E_{15} \lambda_{i}-E_{16}}, \\
& H_{2 i}=\frac{B_{21} H_{1 i}-B_{19} \lambda_{i}}{B_{24}-B_{23} \lambda_{i}-B_{22} \lambda_{i}^{2}} .
\end{aligned}
$$

In view of solution (38), stress components (24)-(26) take the form

$$
\begin{aligned}
& {\left[\sigma_{x x}^{*}, \sigma_{x y}^{*}, \sigma_{y y}^{*}\right](x)} \\
& \quad=\sum_{i=1}^{3}\left[H_{3 i}, H_{4 i}, H_{5 i}\right] M_{i}(m, \omega) e^{-\lambda_{i} x-n x},
\end{aligned}
$$

$$
\text { for } \operatorname{Re}\left(\lambda_{i}\right)>0 \text {, }
$$

where

$$
\begin{aligned}
& H_{3 i}=-\lambda_{i}+\iota m A_{11} H_{1 i}-H_{2 i}, \\
& H_{4 i}=A_{12}\left(\iota m-\lambda_{i} H_{1 i}\right), \\
& H_{5 i}=-A_{11} \lambda_{i}+\iota m H_{1 i}-H_{2 i} .
\end{aligned}
$$

\section{Application: Mechanical Load on the Surface of the Half-Space}

A nonhomogeneous, rotating, magneto-thermoelastic medium, occupying the half-space $x \geq 0$, has been considered. The surface $x=0$ of the half-space is acted upon by a mechanical load as shown in Figure 1. So, the boundary conditions are given by the following.

(i) Mechanical Boundary Conditions

(i) Normal stress component obeys

$$
\sigma_{x x}(0, y, t)=-p(y, t)
$$

where $p(y, t)$ is a given function of $y$ and $t$.

(ii) Tangential stress component vanishes at the surface $x=0$, i.e.,

$$
\sigma_{x y}(0, y, t)=0
$$

(ii) Thermal Boundary Condition

Since, the plane boundary surface $x=0$ is taken to be isothermal, so the thermal boundary condition is the vanishing of temperature $\theta$, i.e.,

$$
\theta(0, y, t)=0
$$

Application of nondimensional parameters and normal mode technique defined in (22) and (31) respectively, transforms the above boundary conditions to the form:

$$
\begin{gathered}
\sigma_{x x}^{*}(x)=-p^{*}, \\
\sigma_{x y}^{*}(x)=0, \\
\theta^{*}(x)=0
\end{gathered}
$$

$$
\text { at } x=0 \text {. }
$$

Taking into account the nondimensional expressions for temperature and stresses from (38) and (40), the above 
boundary conditions reduce to a nonhomogeneous system of three equations, which can be written in matrix form as

$$
\left[\begin{array}{lll}
H_{31} & H_{32} & H_{33} \\
H_{41} & H_{42} & H_{43} \\
H_{21} & H_{22} & H_{23}
\end{array}\right]\left[\begin{array}{l}
M_{1} \\
M_{2} \\
M_{3}
\end{array}\right]=\left[\begin{array}{c}
-p^{*} \\
0 \\
0
\end{array}\right] .
$$

Solution of system (46) provides us the values of $M_{i}(i=$ $1,2,3)$ as follows:

$$
\begin{aligned}
& M_{1}=\frac{\Delta_{1}}{\Delta}, \\
& M_{2}=\frac{\Delta_{2}}{\Delta}, \\
& M_{3}=\frac{\Delta_{3}}{\Delta},
\end{aligned}
$$

where

$$
\begin{aligned}
\Delta & =H_{31} L_{1}-H_{32} L_{2}+H_{33} L_{3}, \\
\Delta_{1} & =-p^{*} L_{1}, \\
\Delta_{2} & =p^{*} L_{2}, \\
\Delta_{3} & =-p^{*} L_{3}, \\
L_{1} & =H_{42} H_{23}-H_{22} H_{43}, \\
L_{2} & =H_{41} H_{23}-H_{21} H_{43}, \\
L_{3} & =H_{41} H_{22}-H_{21} H_{42} .
\end{aligned}
$$

Substitution of (47) into expressions (38) and (40) provides us the following expressions of field variables

$$
\begin{array}{r}
{\left[u_{1}^{*}, u_{2}^{*}, \theta^{*}\right](x)=\frac{1}{\Delta} \sum_{i=1}^{3}\left(1, H_{1 i}, H_{2 i}\right) \Delta_{i} e^{-\lambda_{i} x},} \\
\text { for } \operatorname{Re}\left(\lambda_{i}\right)>0, \\
{\left[\sigma_{x x}^{*}, \sigma_{x y}^{*}, \sigma_{y y}^{*}\right](x)=\frac{1}{\Delta} \sum_{i=1}^{3}\left(H_{3 i}, H_{4 i}, H_{5 i}\right) \Delta_{i} e^{-\lambda_{i} x-n x},} \\
\text { for } \operatorname{Re}\left(\lambda_{i}\right)>0 .
\end{array}
$$

\section{Notable Cases}

7.1. Neglecting Rotational Effect. In the absence of rotation (i.e., $\Omega=0$ ), we shall be left with the relevant problem in a nonhomogeneous, isotropic, magneto-thermoelastic medium in the context of GN theory III. In this limiting case, we get the corresponding expressions of the physical quantities from (49) and (50).

7.2. Neglecting Nonhomogeneity Effect. By setting $n=0$ in (24)-(29), one can get required expressions for different distributions from (49) and (50). In this limiting case, our results coincide with those of Abo-Dahab et al. [29] with appropriate changes in loading and boundary conditions.

\section{Numerical Results and Discussion}

With an aim to illustrate the obtained theoretical results in the preceding section, we now present some numerical results. The following relevant physical constants are taken from Abo-Dahab et al. [29] for a copper-like material:

$$
\begin{aligned}
& \lambda_{0}=7.76 \times 10^{10} \mathrm{~N} \mathrm{~m}^{-2}, \\
& \mu_{0}=3.86 \times 10^{10} \mathrm{~N} \mathrm{~m}^{-2}, \\
& K_{0}=0.6 \times 10^{-2} \mathrm{~W} \mathrm{~m}^{-1} \mathrm{~K}^{-1}, \\
& C_{e}=383.1 \mathrm{~J} \mathrm{~kg}^{-1} \mathrm{~K}^{-1}, \\
& \rho_{0}=8954 \mathrm{~kg} \mathrm{~m}^{-3}, \\
& T_{0}=293 \mathrm{~K}, \\
& \alpha_{t}=1.78 \times 10^{-5} \mathrm{~K}^{-1} .
\end{aligned}
$$

Since $\omega$ is complex quantity, we can write $\omega=\omega_{0}+\iota \omega_{1}$ so that $e^{\omega t}=e^{\omega_{0} t}\left[\cos \left(\omega_{1} t\right)+\iota \sin \left(\omega_{1} t\right)\right]$. So for small values of time we can assume $\omega$ as real (i.e., $\omega=\omega_{0}$ ). The other parameters for numerical computation are taken as $\omega=2, m=2, t=0.1$, $p^{*}=8$, and $y=0.2$.

Figures 2-5 analyze the effect of rotation on the distribution of field variables by considering three different values of angular velocity as $\Omega=0.0$ (solid line), $\Omega=0.5$ (dashed line), and $\Omega=0.9$ (dot-dashed line) with $n=1.0$ and $H_{0}=10^{5}$. Figure 2 explains the spatial variation of the normal displacement component for different values of $\Omega$. The figure shows that the distribution of normal displacement follows a similar trend for all the values of $\Omega$ and dissimilarity lies on the ground of magnitudes. Figure 3 is plotted to depict the variation of normal stress with location $x$ for three different values of $\Omega$. The figure shows that increase in the value of $\Omega$ results in an increase in the numerical values of normal stress. Therefore, angular velocity is having an increasing effect on the profile of normal stress. Variations in tangential stress distribution with spatial coordinate $x$ have been displayed in Figure 4. These variations are having a common starting point of zero magnitude, which is in quite good agreement with the boundary conditions. The figure shows that the tangential stress increases in the beginning and starts decreasing near the point $x=0.23$ and thereafter converges to zero as $x$ increases. Moreover, with increasing $\Omega$, there is an increase in the magnitude of tangential stress distribution. Figure 5 is drawn to observe the effect of angular velocity on the pattern of temperature distribution. As expected, the temperature distribution is having a coincident starting point of zero magnitude for all the values of $\Omega$, which agrees completely with the boundary conditions. It is also manifested from the figure that increasing values of $\Omega$ are having a decreasing effect on the magnitude of temperature variations.

Figures 6-9 illustrate the effect of the nonhomogeneity parameter on the distribution of field variables by setting three different values of nonhomogeneity parameter as $n=0.0$ (solid line), $n=0.5$ (dashed line), and $n=1.0$ (dotdashed line) with $\Omega=0.5$ and $H_{0}=10^{5}$. In Figure 6, we have 


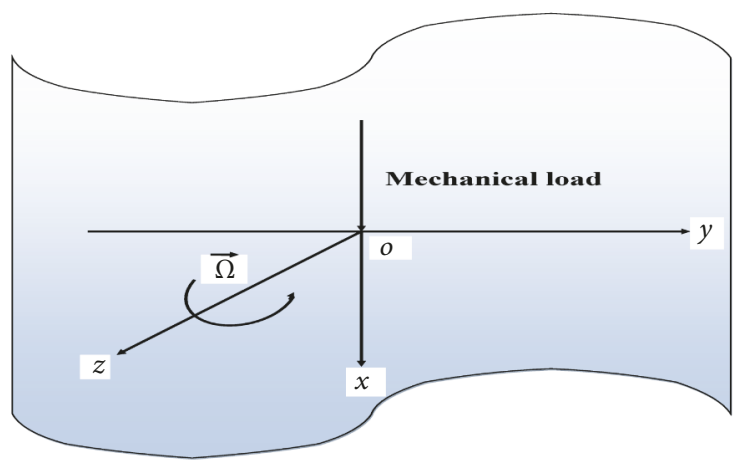

FIGURE 1: Mechanical load over a functionally graded rotating magneto-thermoelastic medium.

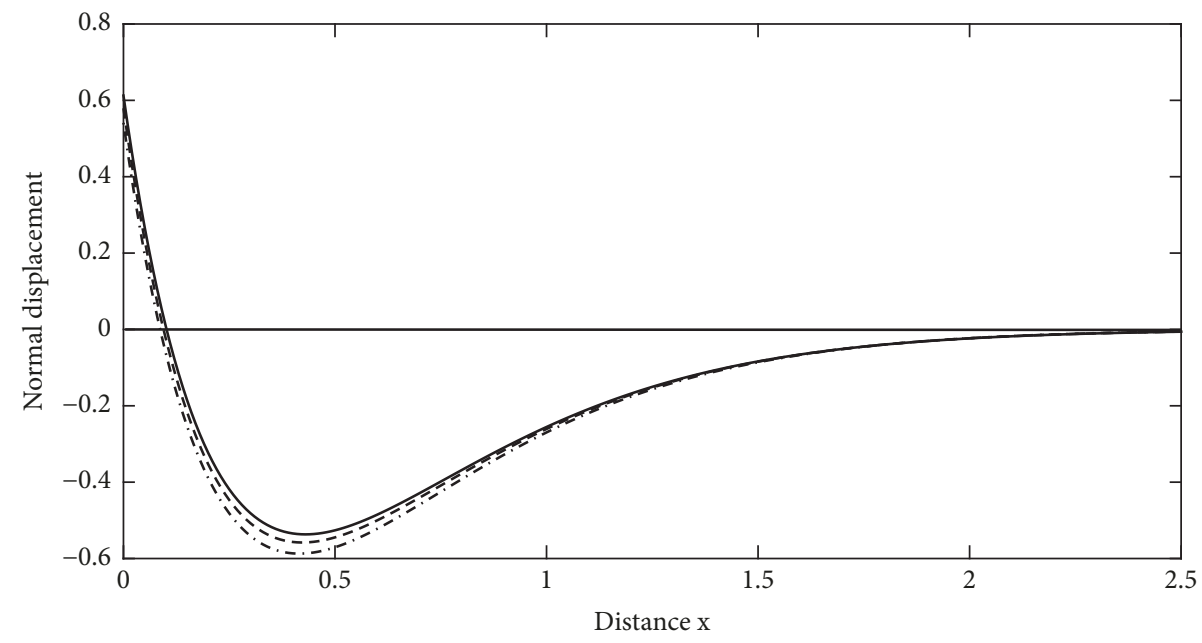

$-\Omega=0.0$

- - $\Omega=0.5$

. . . $\Omega=0.9$

FIGURE 2: Effect of angular velocity on normal displacement distribution.

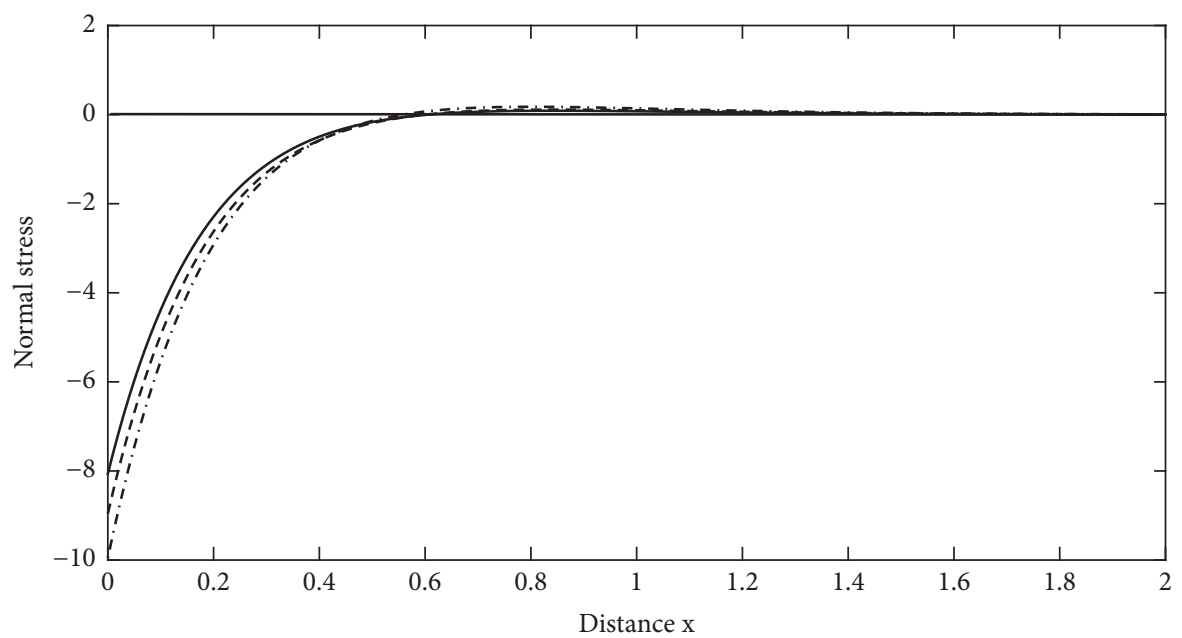

$-\Omega=0.0$

- - $\Omega=0.5$

. . . $\Omega=0.9$

FIGURE 3: Effect of angular velocity on normal stress distribution. 


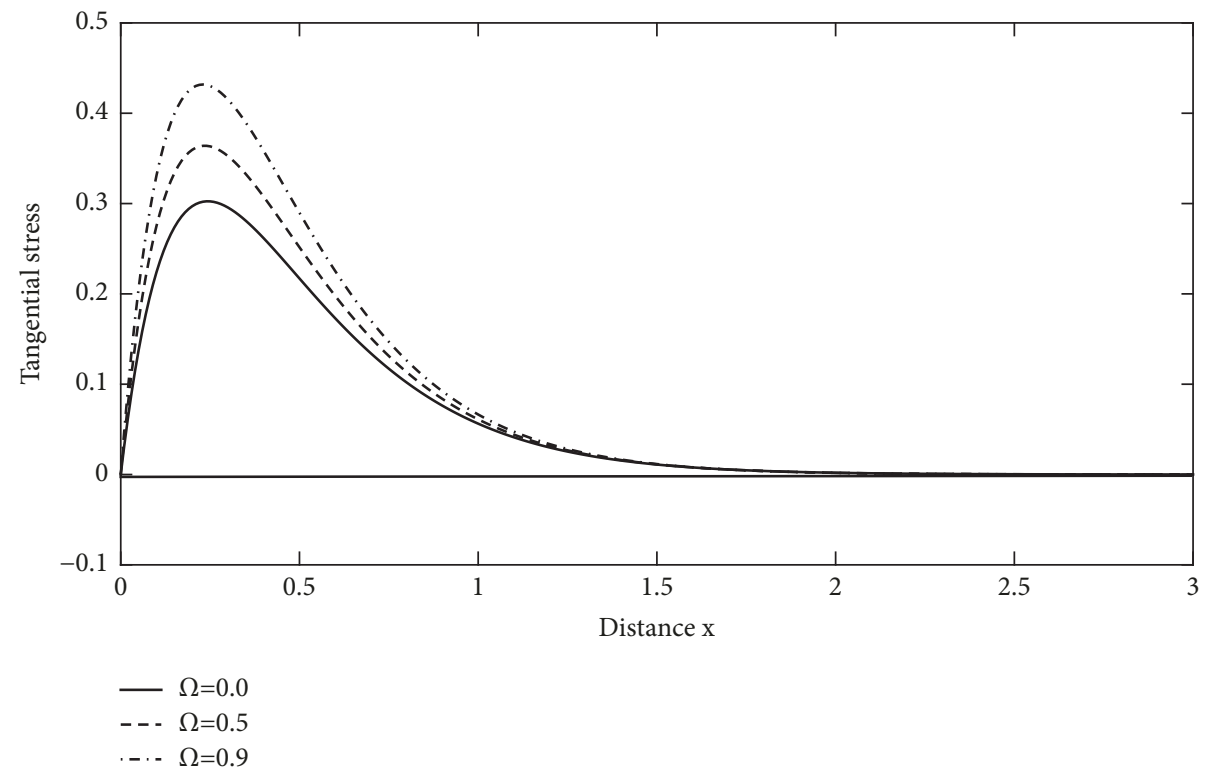

FIGURE 4: Effect of angular velocity on tangential stress distribution.

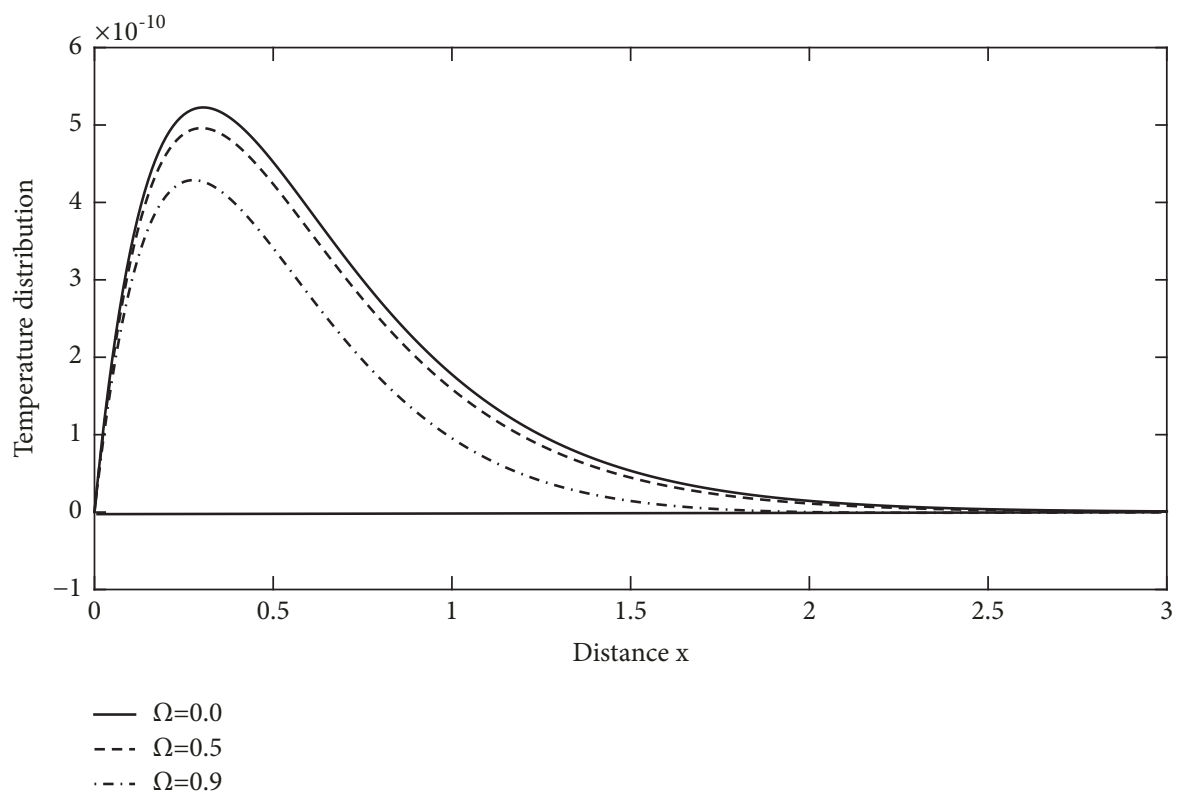

FIGURE 5: Effect of angular velocity on temperature distribution.

shown the spatial variation of normal displacement for different values of $n$. From this figure, it is noted that all the curves have distinct starting points. It can also be noticed from the plot that the displacement distribution is strongly affected by the presence of nonhomogeneity parameter. Figure 7 illustrates the variation of normal stress with distance $x$ for different values of nonhomogeneity parameter $n$. For three different values of nonhomogeneity parameter, $\sigma_{x x}$ starts with value 8.9 (in magnitude). Normal stress distribution exhibits significant sensitivity towards the nonhomogeneity parameter and it is compressive for homogeneous medium. Figure 8 is plotted to show the variations of tangential stress $\sigma_{x y}$ with distance $x$. The plot indicates that the tangential stress field is having a coincident starting point of zero magnitude for all the three cases, which signifies that the boundary conditions are satisfied. It can be seen from the plot that, for homogeneous medium, the behaviour of $\sigma_{x y}$ is totally opposite to that in the nonhomogeneous medium. The difference in magnitudes becomes indistinct along with the passage of time. Figure 9 shows that the temperature starts with a value zero which is completely in agreement with the boundary conditions. It increases in the beginning and starts decreasing in the neighbourhood of $x=0.3$ and converges to zero as $x$ increases. It is also manifested from the figure that 


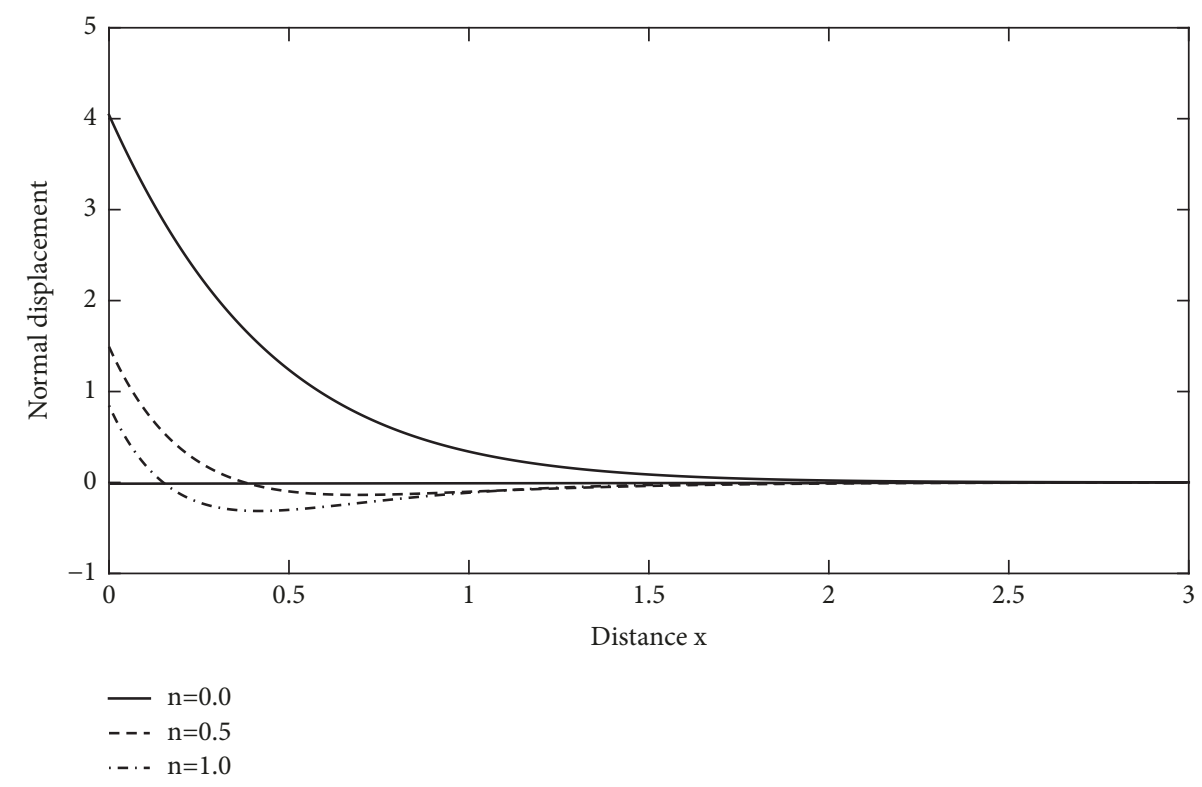

FIGURE 6: Effect of nonhomogeneity parameter on normal displacement distribution.

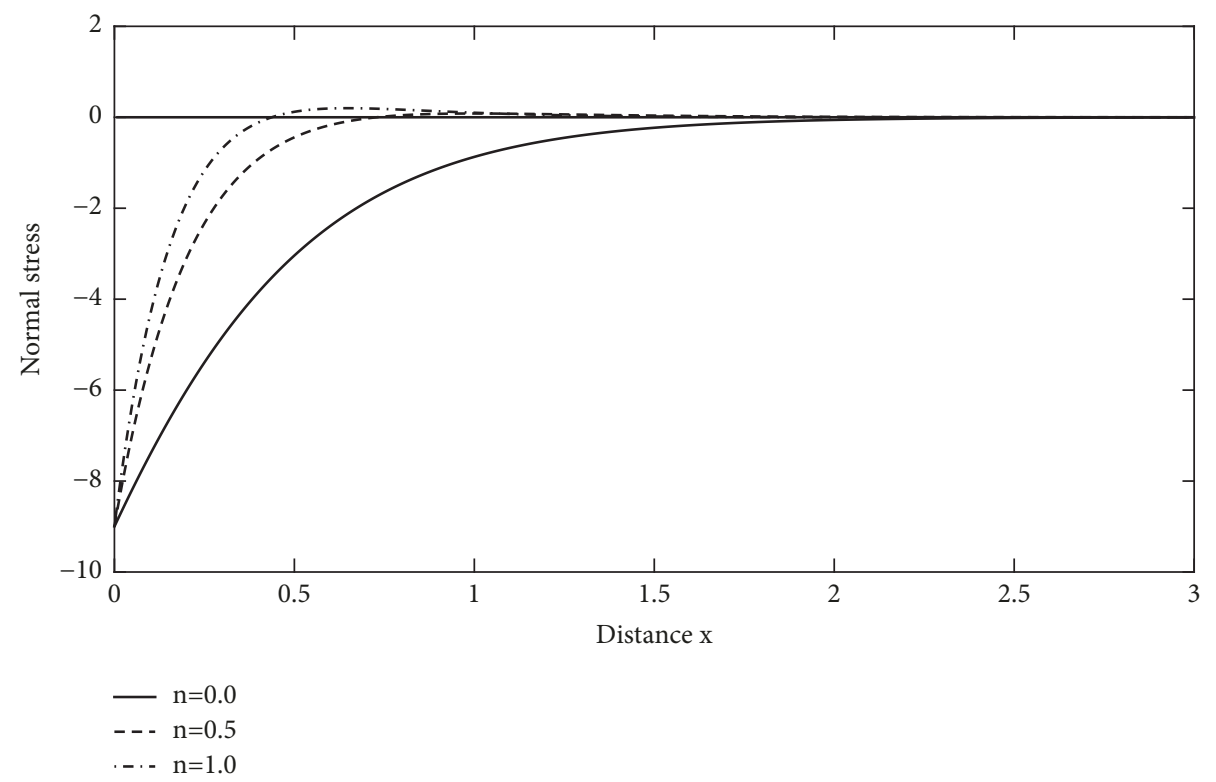

FIGURE 7: Effect of nonhomogeneity parameter on normal stress distribution.

the presence of nonhomogeneity is having a decreasing effect on the magnitude of temperature distribution.

The influence of magnetic field on various field variables is examined in Figures 10-13. Figure 10 shows the transient effect of applied mechanical load on normal displacement distribution, in the medium with a magnetic field (solid line) and without magnetic field (dashed line). Solution curves for both the cases follow a similar pattern of variations with the difference in magnitudes. It can be noted that magnetic field has a noticeable impact on displacement distribution. Figure 11 describes the variation of normal stress with location $x$. The plot shows that stress $\sigma_{x x}$ is having a coincident initial point for both the cases and diminution of the magnitude takes place as the distance from the boundary increases. Figure 12 represents the spatial variation of tangential stress. It can be seen from the plot that the values of tangential stress for both the cases increase in the beginning and start decreasing in the neighbourhood of $x=0.2$ and approach to zero with increasing $x$. Figure 13 displays the variation of temperature field with distance $x$. In this figure, all the curves have coincident beginning point with value zero that leads to satisfy the boundary conditions. The amplitude of vibrations 


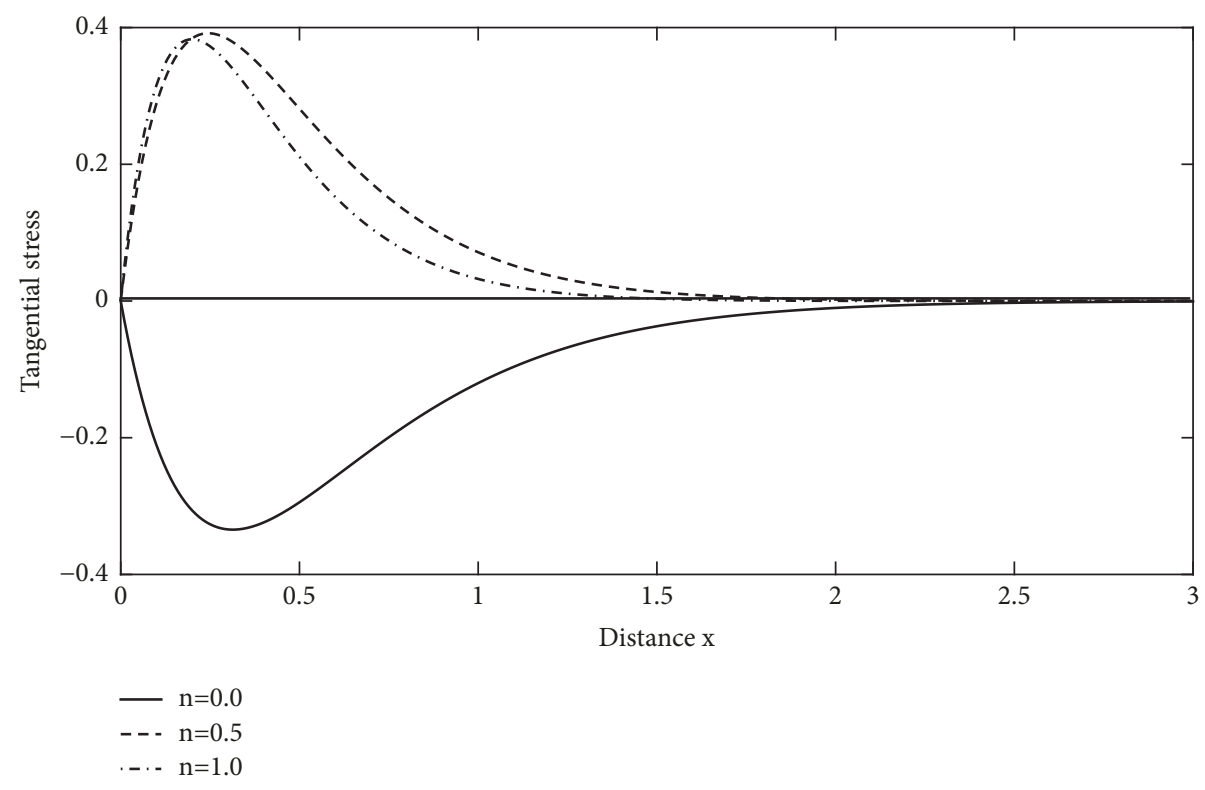

FIGURE 8: Effect of nonhomogeneity parameter on tangential stress distribution.

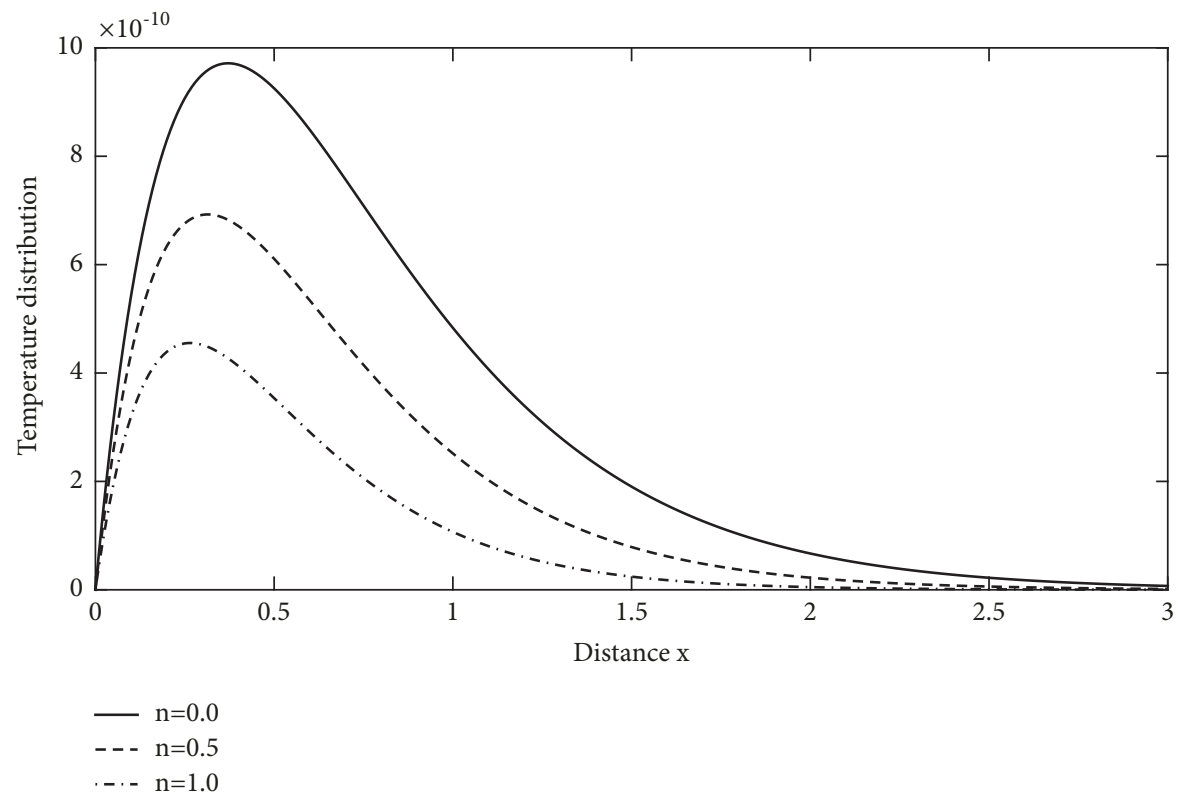

FIGURE 9: Effect of nonhomogeneity parameter on temperature distribution.

of solution curves for temperature field gets suppressed in the absence of magnetic field.

The 3D plots showing normal displacement distribution, normal stress distribution, tangential stress distribution, and temperature distribution are shown in Figures 14-17 for a wide range of $x(0 \leq x \leq 4)$ and for a wide range of dimensionless time $t(0 \leq t \leq 0.4)$. Figure 14 describes the variation of normal displacement with distance $x$ and with time $t$. From this figure, it can be seen that the dimensionless time $t$ plays an important role on the distribution of normal displacement. Figure 15 represents the variation in the values of normal stress for a wide range of $x$. Numerical values of normal stress decrease as the distance $x$ increases, while for time $t$, an increase in the values takes place. Figure 16 has been plotted to show the profile of tangential stress distribution. The tangential stress starts with a zero value which is completely in agreement with the boundary conditions prescribed. Figure 17 represents the distribution of temperature with distance $x$ and with time $t$. The temperature distribution is behaving like an increasing function in the range $0.0 \leq x \leq 0.2$ and for the rest of the domain it decreases and reaches a steady state about the point $x=2.7$. 


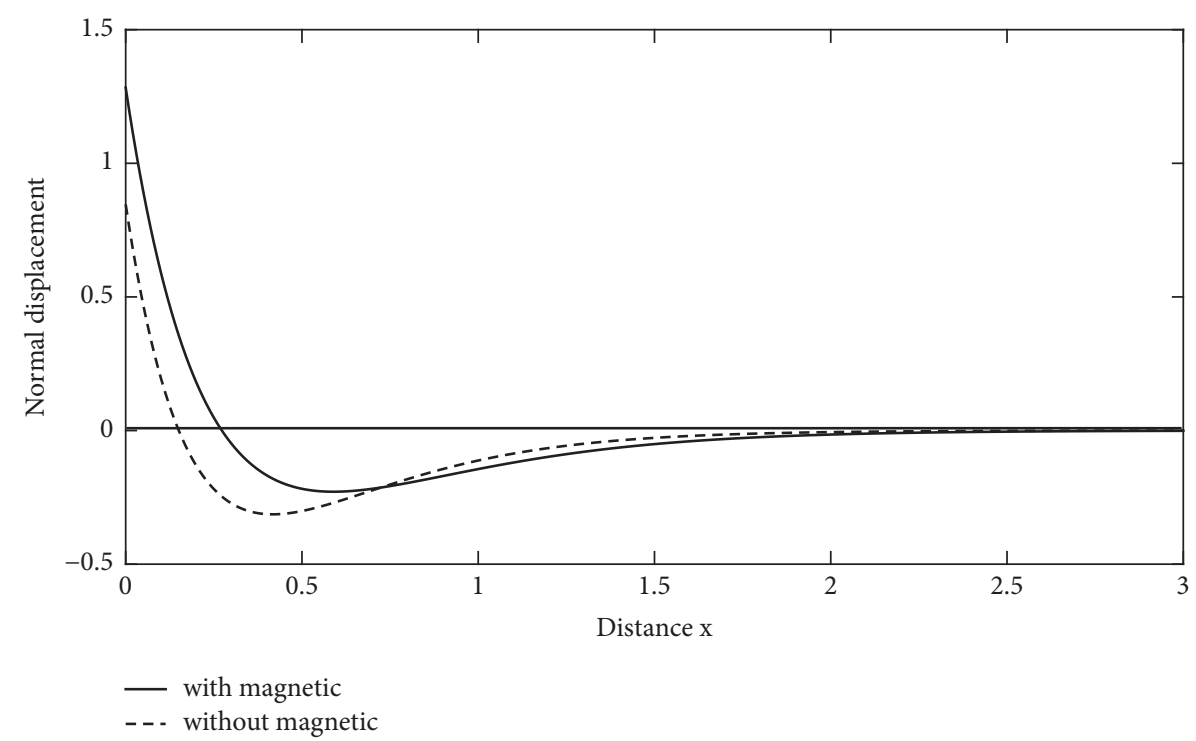

FIGURE 10: Effect of magnetic field on normal displacement.

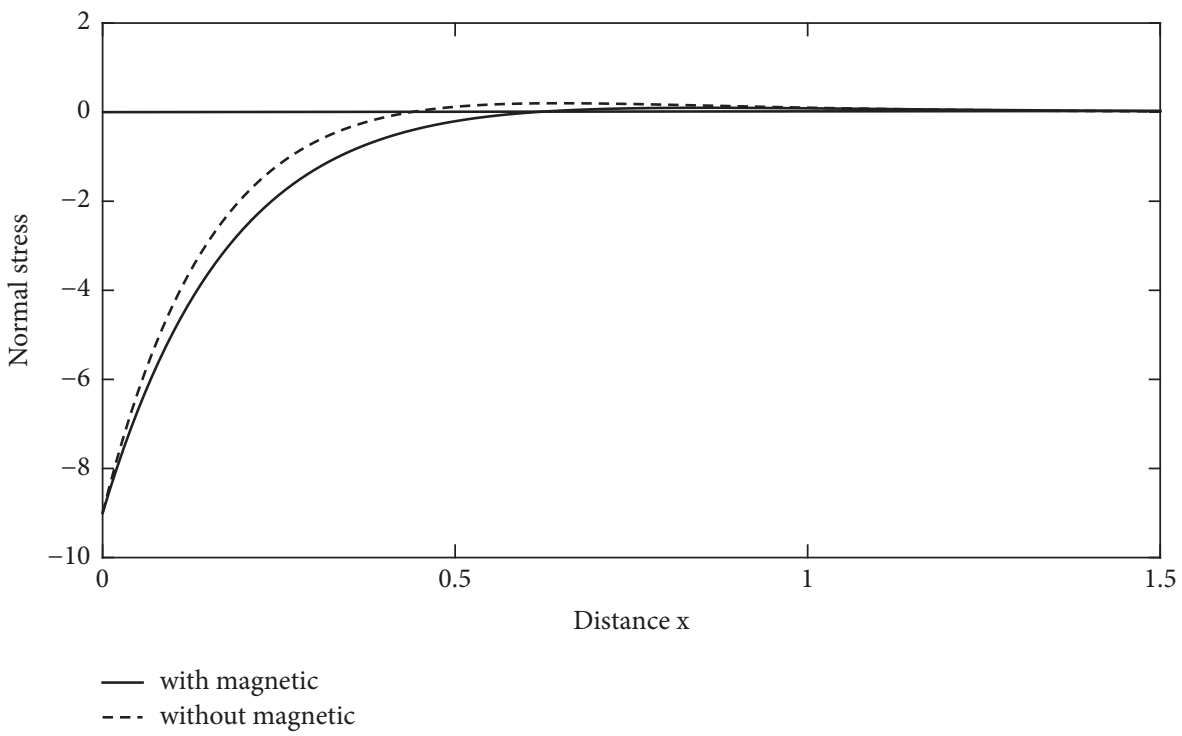

FIGURE 11: Effect of magnetic field on normal stress distribution.

\section{Concluding Remarks}

The investigation under consideration provides a mathematical model to obtain the behaviour of normal displacement, stresses, and temperature in a nonhomogeneous, isotropic, rotating, magneto-thermoelastic medium within the framework of G-N model III, by using normal mode technique. Theoretical and numerical results reveal that the parameters, namely, rotation, nonhomogeneity parameter, and magnetic field, have significant effects on the considered physical variables. Analysis of graphs permits the following concluding remarks: (i) As expected, the values of all the physical quantities converge to zero as the distance $x$ increases and from the distribution of all physical quantities, it can be found that wave type heat propagates through the medium.

(ii) It is apparent from figures that the rotational speed has an increasing effect on the profiles of normal stress and tangential stress but it has a decreasing effect on the profile of temperature.

(iii) The presence of nonhomogeneity is having a decreasing effect on the magnitude of temperature distribution while it has a mix effect on the remaining 


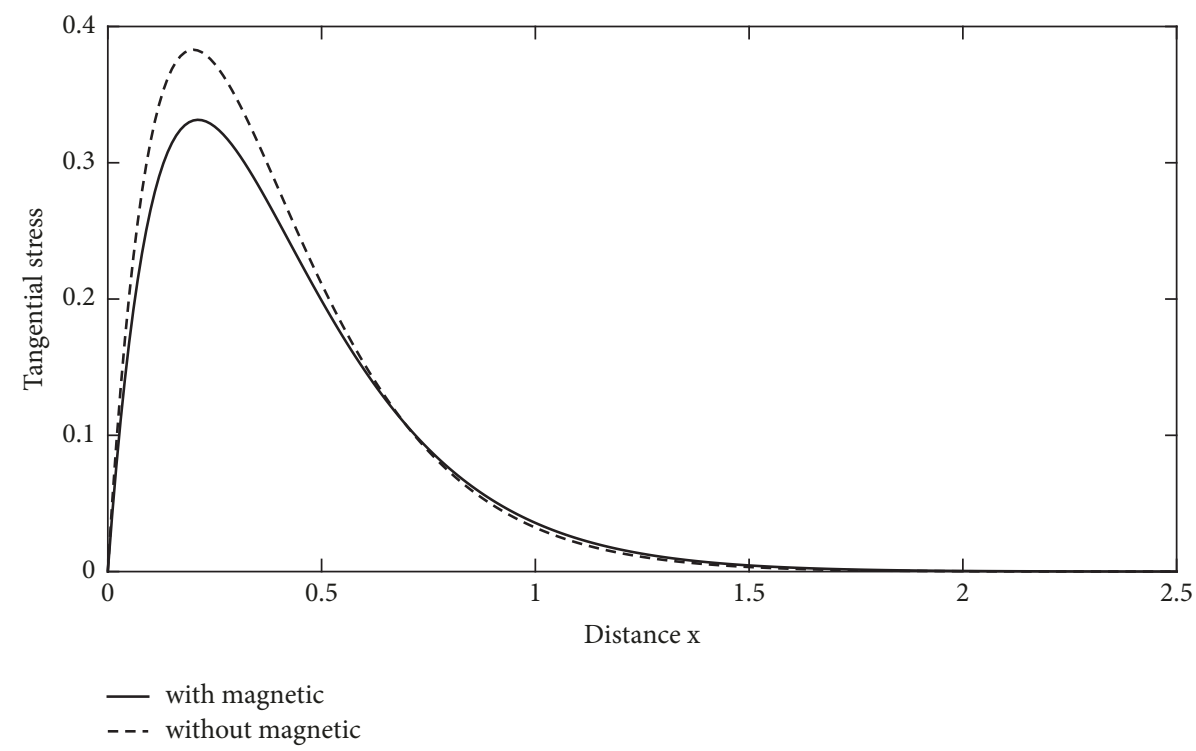

FIGURE 12: Effect of magnetic field on tangential stress distribution.

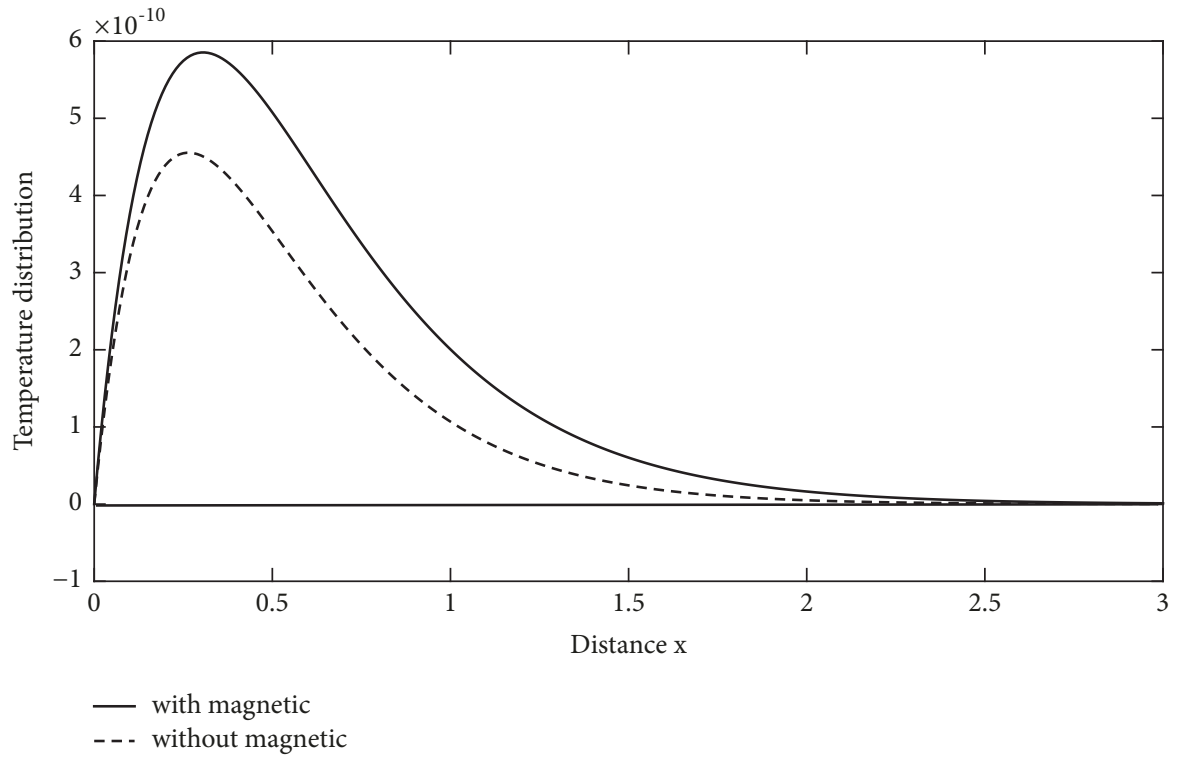

FIGURE 13: Effect of magnetic field on temperature distribution.

field variables. Therefore, while designing FGMs, the effect of nonhomogeneity should be taken into consideration.

(iv) The presence of magnetic field plays a significant role in the distribution of all physical variables.

(v) The method adopted here is applicable to a wide range of problems in thermodynamics and thermoelasticity. It can be employed to boundary-layer problems which are described by linearized Navier-Stokes equations in electro-hydrodynamics.
The above study is of geophysical interest and finds applications in mechanical engineering, industrial sectors, and seismology. The results presented in this paper will prove useful for scientists in material science and designers of new materials as well as for those working on the development of magneto-thermoelasticity. Electro-magneto composite materials have applications in sensors, actuators, ultrasonic imaging devices, and many other emerging components. Magneto-thermoelasticity has drawn the attention of many engineers, because of its wide use in diverse areas, especially, geophysics for determining the effect of earth's magnetic field on seismic waves, development of a highly 


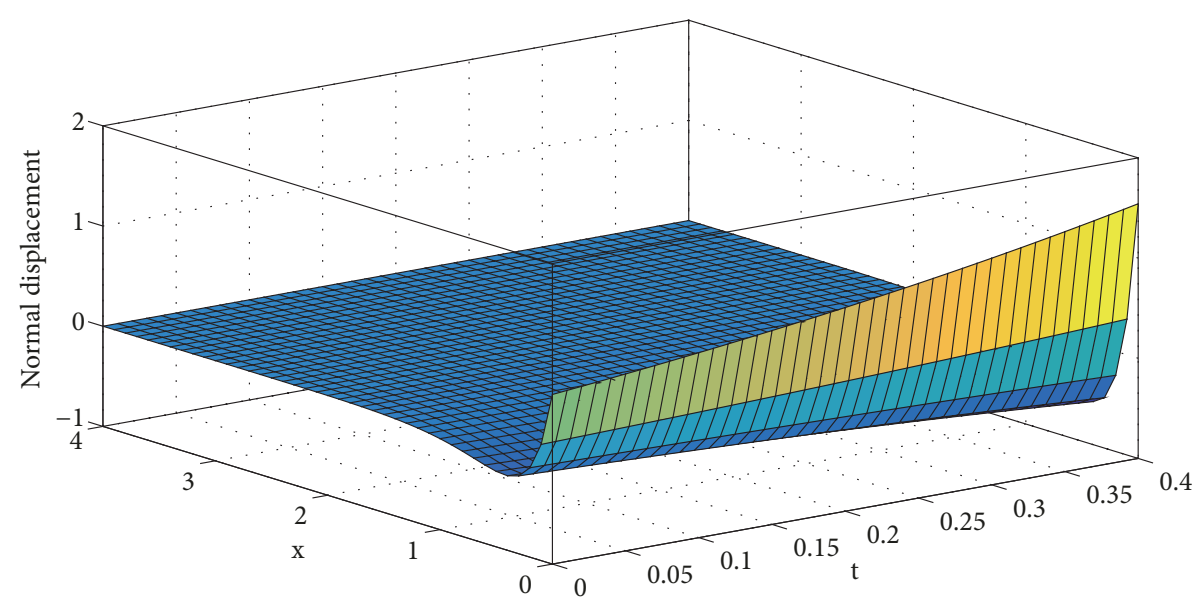

FIgURE 14: Profile of normal displacement at $n=1.0, H_{0}=10^{5}$, and $\Omega=0.5$.

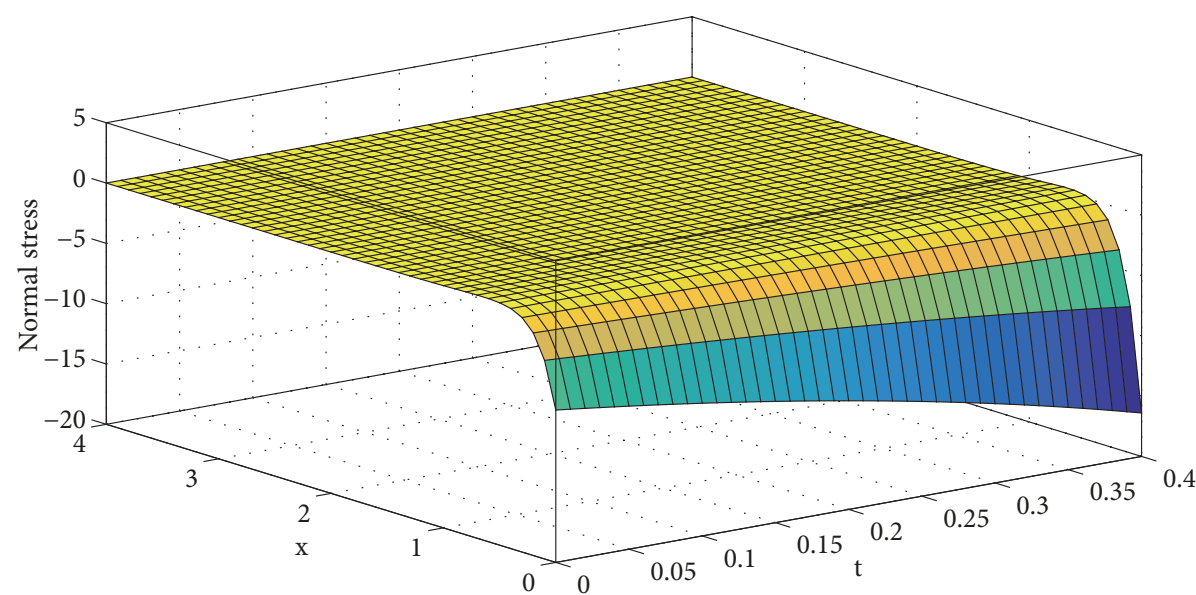

FigURE 15: Profile of normal stress distribution at $n=1.0, H_{0}=10^{5}$, and $\Omega=0.5$.

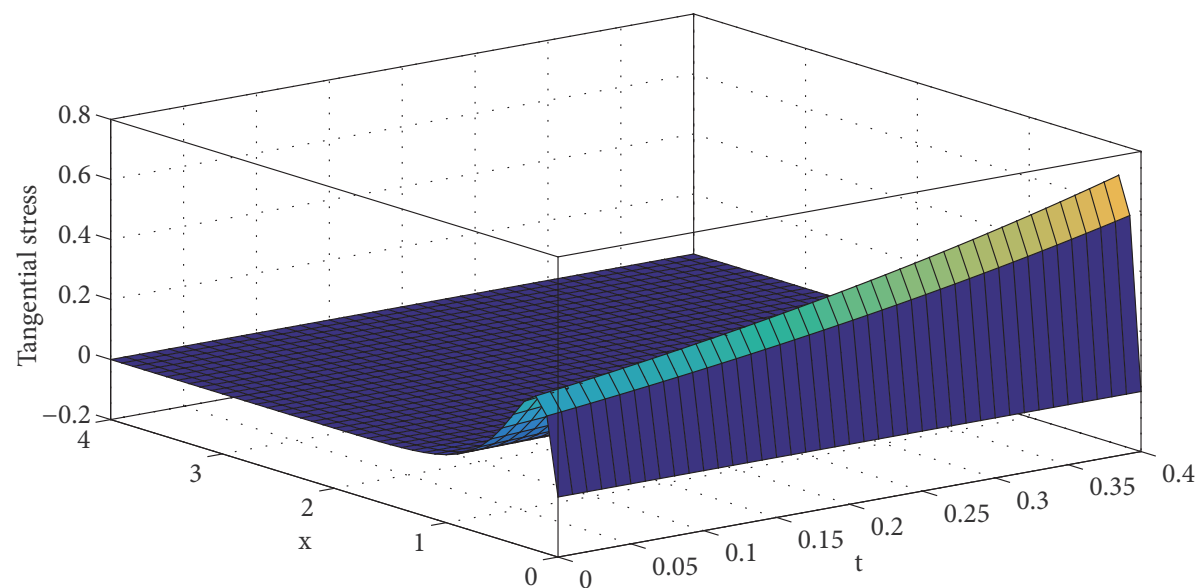

FIGURE 16: Profile of tangential stress distribution at $n=1.0, H_{0}=10^{5}$, and $\Omega=0.5$.

sensitive superconducting magneto-meter, electrical power engineering, etc. FGMs are broadly used in the biomedical application as medical implants, because they are designed to mimic the human organs, which are FGMs in nature. These types of materials are also used in pressure vessels and pipes.

\section{Data Availability}

The data used in this study are included within the article in the section "Numerical Results and Discussion".

\section{Conflicts of Interest}

No potential conflicts of interest were reported by the authors. 


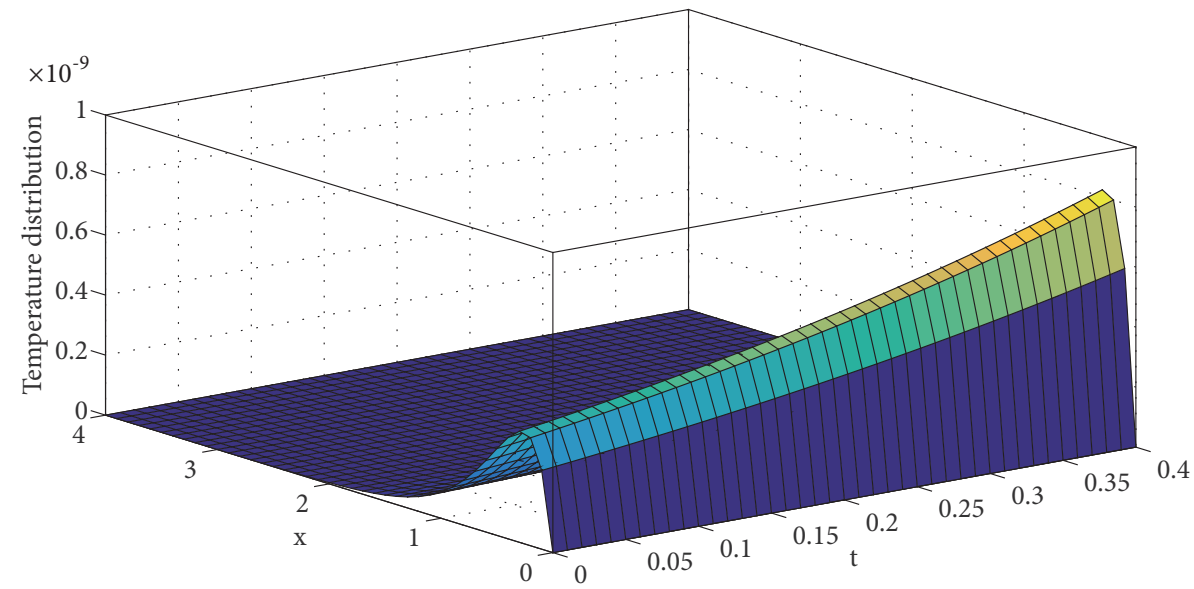

FIGURE 17: Profile of temperature distribution at $n=1.0, H_{0}=10^{5}$, and $\Omega=0.5$.

\section{Acknowledgments}

One of the authors Mr. Ankush Gunghas is thankful to Haryana State Council for Science and Technology (HSCST) for the financial support.

\section{References}

[1] M. A. Biot, "Thermoelasticity and irreversible thermodynamics," Journal of Applied Physics, vol. 27, pp. 240-253, 1956.

[2] H. W. Lord and Y. Shulman, "A generalized dynamical theory of thermoelasticity," Journal of the Mechanics and Physics of Solids, vol. 15, no. 5, pp. 299-309, 1967.

[3] A. E. Green and K. A. Lindsay, "Thermoelasticity, Journal of Elasticity, vol. 2, no. 1, pp. 1-7, 1972.

[4] A. E. Green and P. M. Naghdi, "A re-examination of the basic postulates of thermomechanics," Proceedings of the Royal Society A Mathematical, Physical and Engineering Sciences, vol. 432, pp. 171-194, 1991.

[5] A. E. Green and P. M. Naghdi, "On undamped heat waves in an elastic solid," Journal of Thermal Stresses, vol. 15, no. 2, pp. 253-264, 1992.

[6] A. E. Green and P. M. Naghdi, "Thermoelasticity without energy dissipation," Journal of Elasticity, vol. 31, no. 3, pp. 189-208, 1993.

[7] M. A. Ezzat, "Generation of generalized magnetothermoelastic waves by thermal shock in a perfectly conducting half-space," Journal of Thermal Stresses, vol. 20, no. 6, pp. 617-633, 1997.

[8] M. A. Ezzat and M. I. Othman, "State-space approach to generalized magnetothermoelasticity with thermal relaxation in a medium of perfect conductivity," Journal of Thermal Stresses, vol. 25, no. 5, pp. 409-429, 2002.

[9] M. I. A. Othman, S. M. Said, and N. Sarker, "Effect of hydrostatic initial stress on a fiber-reinforced thermoelastic medium with fractional derivative heat transfer," Multidiscipline Modeling in Materials and Structures, vol. 9, no. 3, pp. 410-426, 2013.

[10] S. Deswal and K. K. Kalkal, "Plane waves in a fractional order micropolar magneto-thermoelastic half-space," Wave Motion, vol. 51, no. 1, pp. 100-113, 2014.

[11] S. Deswal, S. S. Sheoran, and K. K. Kalkal, "The effect of magnetic field and initial stress on fractional order generalized thermoelastic half-space," Journal of Mathematics, vol. 2013, Article ID 489863, 11 pages, 2013.
[12] M. I. A. Othman and E. E. M. Eraki, "Generalized magnetothermoelastic half-space with diffusion under initial stress using three-phase-lag model," Mechanics Based Design of Structures and Machines, vol. 45, no. 2, pp. 145-159, 2017.

[13] C. Xiong and Y. Guo, "Electromagneto-thermoelastic diffusive plane waves in a half-space with variable material properties under fractional order thermoelastic diffusion," International Journal of Applied Electromagnetics and Mechanics, vol. 53, no. 2, pp. 251-269, 2017.

[14] M. Schoenberg and D. Censor, "Elastic waves in rotating media," Quarterly of Applied Mathematics, vol. 31, no. 1, pp. 115-125, 1973.

[15] S. K. Roy Chaudhuri and L. Debnath, "Magneto-thermoelastic plane waves in rotating media," International Journal of Engineering Science, vol. 21, no. 2, pp. 155-163, 1983.

[16] S. K. Roy Choudhuri and S. Mukhopadhyay, "Effect of rotation and relaxation times on plane waves in generalized thermo-visco-elasticity," International Journal of Mathematics and Mathematical Sciences, vol. 23, pp. 497-505, 2000.

[17] P. Ailawalia and N. S. Narah, "Effect of rotation in generalized thermoelastic solid under the influence of gravity with an overlying infinite thermoelastic fluid," Applied Mathematics and Mechanics-English Edition, vol. 30, no. 12, pp. 1505-1518, 2009.

[18] A. E. Abouelregal and A. M. Zenkour, "The effect of fractional thermoelasticity on a two-dimensional problem of a mode I crack in a rotating fiber-reinforced thermoelastic medium," Chinese Physics B, vol. 22, no. 1-8, Article ID 108102, 2013.

[19] R. Kumar, K. D. Sharma, and S. K. Garg, "Effect of two temperatures on reflection coefficient in micropolar thermoelastic with and without energy dissipation media," Advances in Acoustics and Vibration, vol. 2014, Article ID 846721, 11 pages, 2014.

[20] M. I. A. Othman, W. M. Hasona, and E. M. Abd-Elaziz, "Effect of rotation on micropolar generalized thermoelasticity with two temperatures using a dual-phase lag model," Canadian Journal of Physics, vol. 92, no. 2, pp. 149-158, 2014.

[21] S. M. Said, Y. D. Elmaklizi, and M. I. A. Othman, "A twotemperature rotating-micropolar thermoelastic medium under influence of magnetic field," Chaos, Solitons \& Fractals, vol. 97, pp. 75-83, 2017.

[22] A. E. Abouelregal and S. M. Abo-Dahab, "A two-dimensional problem of a mode-I crack in a rotating fibre-reinforced isotropic thermoelastic medium under dual-phase-lag model," Sadhana, vol. 43, no. 1, article no. 13, 2018. 
[23] N. S. Hussien and F. S. Bayones, "Effect of rotation on Rayleigh waves in a fiber-reinforced solid anisotropic magneto-thermoviscoelastic media," Mechanics of Advanced Materials and Structures, pp. 1-8, 2018.

[24] J. Aboudi, M.-J. Pindera, and S. M. Arnold, "Thermo-inelastic response of functionally graded composites," International Journal of Solids and Structures, vol. 32, no. 12, pp. 1675-1710, 1995.

[25] A. M. Abd-Alla, S. M. Abo-Dahab, T. A. Al-Thamali, and S. R. Mahmoud, "Influence of the rotation and gravity field on stonely waves in a non-homogeneous orthotropic elastic medium," Journal of Computational and Theoretical Nanoscience, vol. 10, no. 2, pp. 297-305, 2013.

[26] I. A. Abbas and A. M. Zenkour, "LS model on electro-magnetothermoelastic response of an infinite functionally graded cylinder," Composite Structures, vol. 96, pp. 89-96, 2013.

[27] I. A. Abbas, "Nonlinear transient thermal stress analysis of thick-walled FGM cylinder with temperature-dependent material properties," Meccanica, vol. 49, no. 7, pp. 1697-1708, 2014.

[28] J. D. Kraus, Electromagnetics, McGraw-Hill Inc, USA, 1984.

[29] S. M. Abo-Dahab, A. M. Abd-Alla, and A. J. Alqarni, "A twodimensional problem with rotation and magnetic field in the context of four thermoelastic theories," Results in Physics, vol. 7, pp. 2742-2751, 2017. 


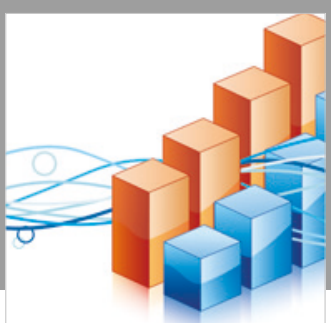

Advances in

Operations Research

\section{-n-m}
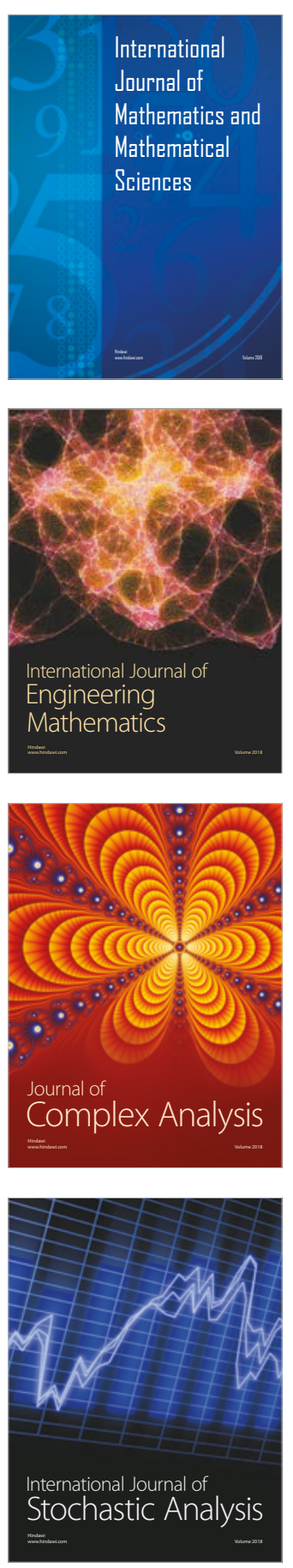
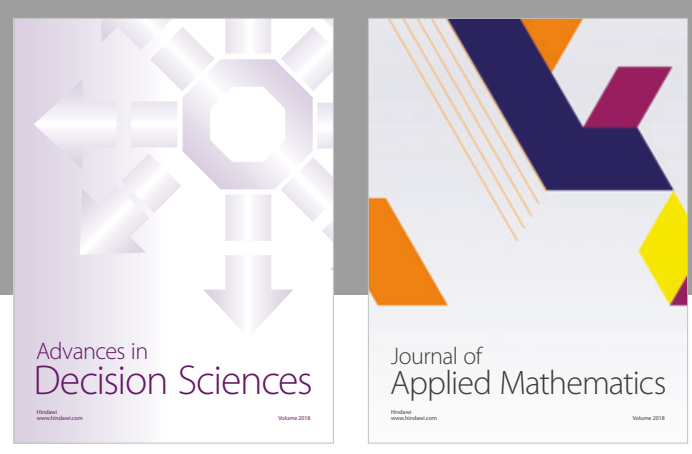

Journal of

Applied Mathematics
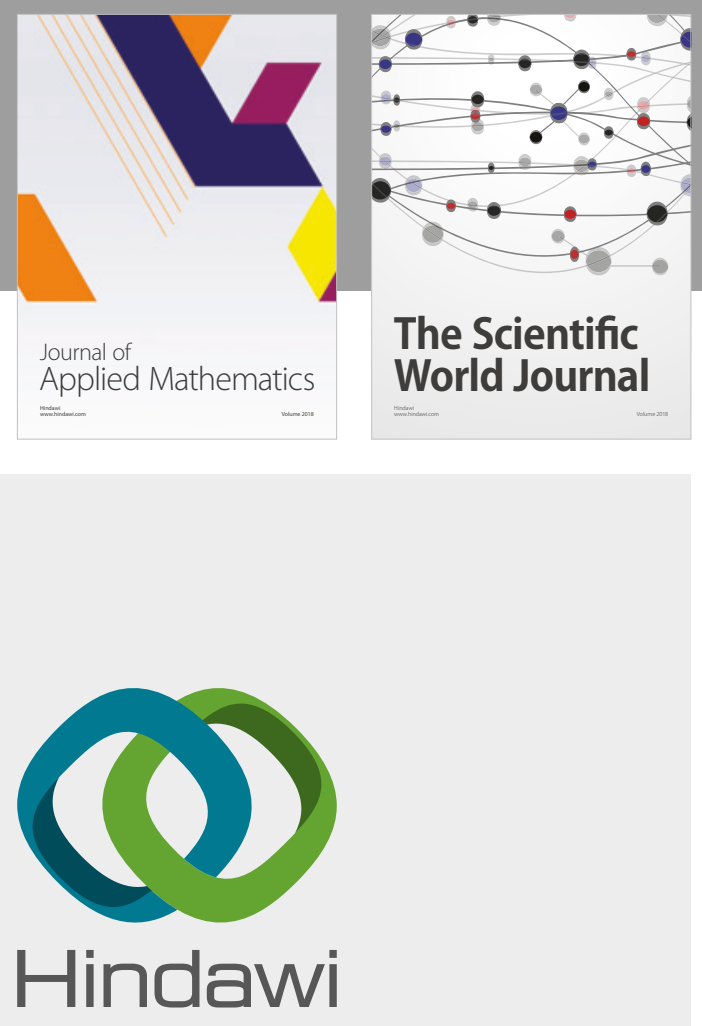

Submit your manuscripts at

www.hindawi.com

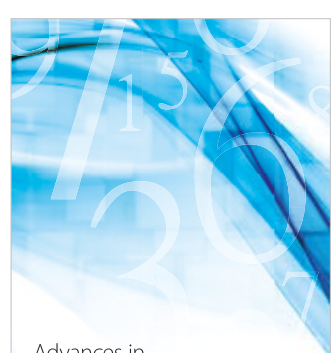

Advances in
Numerical Analysis
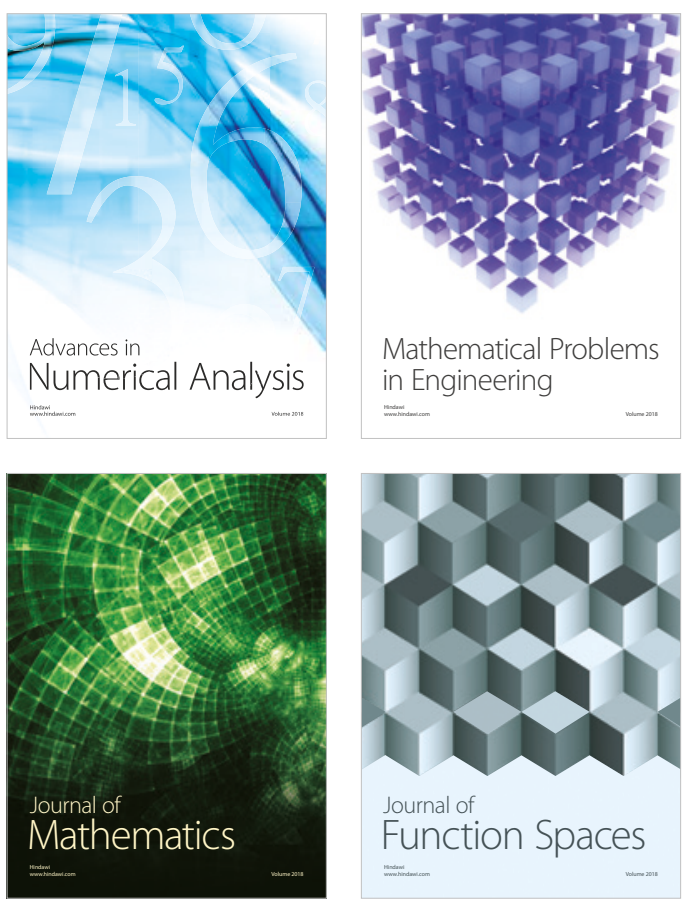

Mathematical Problems in Engineering

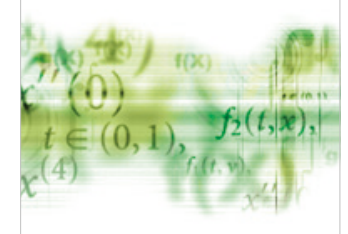

International Journal of

Differential Equations

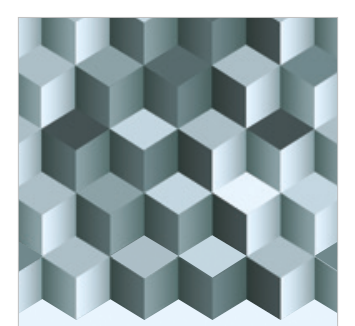

Journal of

Function Spaces

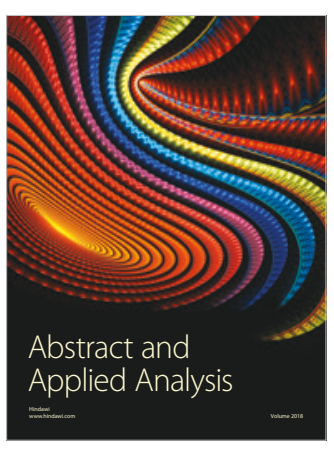

The Scientific

World Journal

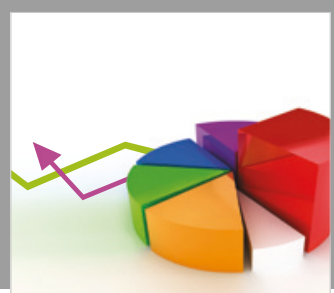

Journal of

Probability and Statistics
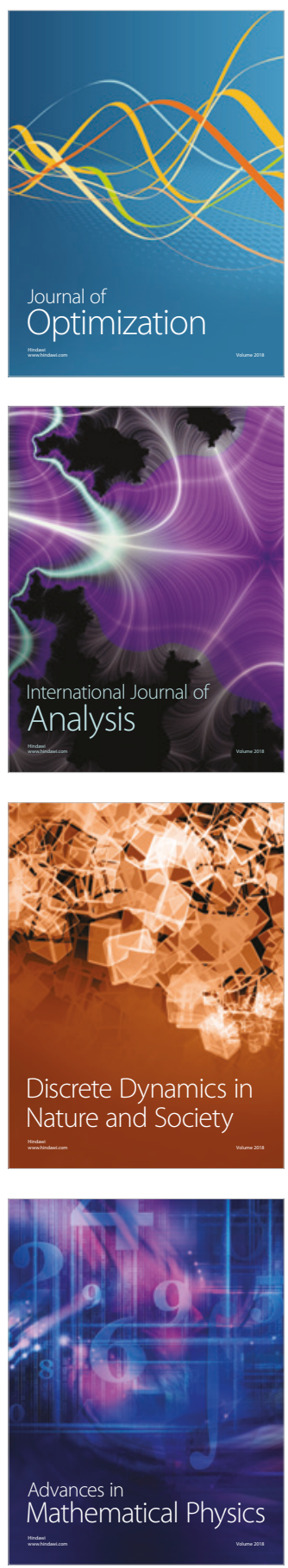